\title{
Developing an International Combined Applied Surgical Science and Wet Lab Simulation Course as an Undergraduate Teaching Model
}

\author{
Michail Sideris, ${ }^{1}$ Apostolos Papalois, ${ }^{2}$ Georgios Tsoulfas, ${ }^{3}$ \\ Sanjib Majumder, ${ }^{4,5}$ Konstantinos Toutouzas, ${ }^{6}$ Efstratios Koletsis, ${ }^{7}$ \\ Panagiotis Dedeilias, ${ }^{8}$ Nikolaos Lymperopoulos, ${ }^{4}$ Savvas Papagrigoriadis, 9 \\ Vassilios Papalois, ${ }^{10,11}$ and Georgios Zografos ${ }^{6,12}$ \\ ${ }^{1}$ King's College Hospital NHS Foundation Trust, Denmark Hill, London SE5 9RS, UK \\ ${ }^{2}$ Experimental Research Centre of ELPEN, 19009 Pikermi, Greece \\ ${ }^{3}$ Aristotle University of Thessaloniki, 54124 Thessaloniki, Greece \\ ${ }^{4}$ Pinderfields Hospital, NHS, Wakefield, West Yorkshire WF1 4DG, UK \\ ${ }^{5}$ University of Leeds, Leeds LS2 9JT, UK \\ ${ }^{6}$ University of Athens, Goudi, 11527 Athens, Greece \\ ${ }^{7}$ University of Patras, 26500 Rio, Greece \\ ${ }^{8}$ Evangelismos Hospital, 10676 Athens, Greece \\ ${ }^{9}$ King's College London, Strand WC2R 2LS, UK \\ ${ }^{10}$ Hammersmith Hospital, London W12 OHS, UK \\ ${ }^{11}$ Imperial College London, London SW7 2AZ, UK \\ ${ }^{12}$ Hippocration University Hospital, 11528 Athens, Greece
}

Correspondence should be addressed to Michail Sideris; msideris@nhs.net

Received 11 June 2015; Accepted 5 October 2015

Academic Editor: Moritz Wente

Copyright (C) 2015 Michail Sideris et al. This is an open access article distributed under the Creative Commons Attribution License, which permits unrestricted use, distribution, and reproduction in any medium, provided the original work is properly cited.

\begin{abstract}
Background. Essential Skills in the Management of Surgical Cases (ESMSC) is an international, animal model-based course. It combines interactive lectures with basic ex vivo stations and more advanced wet lab modules, that is, in vivo dissections and Heart Transplant Surgery on a swine model. Materials and Methods. Forty-nine medical students (male, $N=27$, female $N=22$, and mean age $=23.7$ years) from King's College London (KCL) and Greek Medical Schools attended the course. Participants were assessed with Direct Observation of Procedural Skills (DOPS), as well as Multiple Choice Questions (MCQs). Paired $t$-test associations were used to evaluate whether there was statistically significant improvement in their performance. Aim. To evaluate the effectiveness of a combined applied surgical science and wet lab simulation course as a teaching model for surgical skills at the undergraduate level. Results. The mean MCQ score was improved by 2.33/32 $(P<0.005)$. Surgical skills competences, as defined by DOPS scores, were improved in a statically significant manner $(P<0.005$ for all paired $t$-test correlations). Conclusions. ESMSC seems to be an effective teaching model, which improves the understanding of the surgical approach and the basic surgical skills. In vivo models could be used potentially as a step further in the Undergraduate Surgical Education.
\end{abstract}

\section{Introduction}

Animal model-based simulation has been used for training purposes throughout the time. Most of the current wet lab simulation models have been used for advanced postgraduate training [1-6]. The overall outcome of these courses seems to be satisfactory $[2,3]$. Despite the fact that most of these courses reflect advanced training, recently there are some 
emerging new ones that may be appropriate for undergraduates $[7,8]$.

ESMSC is a two-day international course, which combines applied surgical science lectures with wet lab in vivo and ex vivo simulation skills' modules on a swine model. The course curriculum has been organized effectively in three main cores. The first core refers to four basic science workshops (BScCI), which contain interactive teaching, on the principles of shock and fundamental interpretation of arterial blood gases (ABGs), electrocardiography (ECG), as well as principles in the management of fluids and analgesia. The second core includes case-based interactive lectures (CbCII) on main surgical specialties, which aim to familiarize the participants with a common pattern of approach. This refers to a safety pyramid (Figure 6), which implies a systematic approach for every surgical case. The third core refers to the in vivo and ex vivo wet lab simulation modules (SkCIII), which occupy $50 \%$ of the course curriculum. The novelty of our model lies in the combination of basic knowledge that is required to deal with a patient, that is, fluid resuscitation, ECG, Acid-Base Balance (Basic Science Core), with the principles of a systematic, methodical and safe approach of every surgical patient (Case-Based Lectures and safety pyramid, Figure 6), and the acquisition of fundamental skills required for many common procedures, that is, suturing, IV access, Wound Debridement, chest drain insertion, and basic dissections (Skills' Core). The second unique part of our curriculum is the exposure in some promising in vivo experience, which hopefully drives students' initiative and at the same time expands their ability to effectively perform basic procedures and assist in theatre. Also, watching step by step a live heart transplant would be the highest quality teaching towards the understanding and the consolidation of cardiopulmonary physiology principles. Despite being deemed as an advanced module, it still maintains its teaching and mentorship value even for medical students. The overall idea is to create a curriculum, which will serve as a preparatory step for delivering a generation of well-motivated and efficient future junior trainees in surgery.

In the first in vivo model, participants are taught how to insert a chest tube, demonstrate basic chest anatomy, dissect the abdominal wall, repair primarily perforated bowel tissue, perform a Diagnostic Peritoneal Lavage (DPL), and achieve haemostasis on an actual liver laceration. The second in vivo experiment uses a second anaesthetized pig, in order to demonstrate the principles of extracorporeal circulation. Participants actively assist an experienced team of senior surgeons and enjoy real time teaching on the fundamentals of Heart and Transplant Surgery.

Ex vivo stations include a basic suturing station, where participants are instructed by a team of Plastic Surgeons (SpR and above) on how to perform (and aided in doing so) interrupted, subcuticular, and mattress sutures on swine skin flaps. A balloon is supporting the flap on top of a box, simulating the peritoneum. Another station includes intravenous (IV) access skills and the Seldinger technique for central lines. The third ex vivo station refers to Wound Debridement of swine flaps and primary closure of lacerations. Finally, the last station consists of Open Reduction Internal Fixation

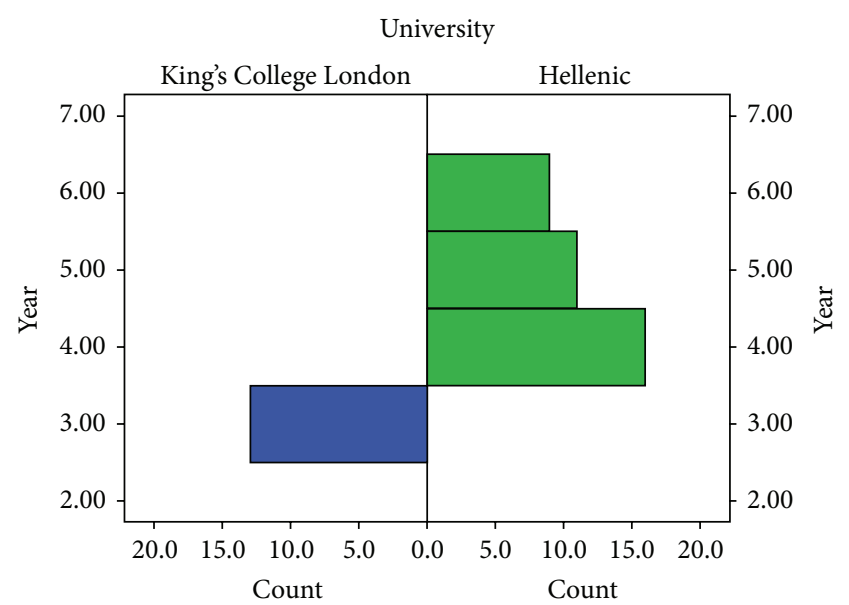

FIGURE 1: Distribution of participants in year of studies.

(ORIF) of hand fractures, where participants have the chance to familiarize themselves in the relevant technique.

For the purposes of the course, a detailed manual has been published (Scientific Publications Parisianou, ISBN: 978-960583-063-2), containing 30 chapters from King's College in London and Greek Universities.

\section{Materials and Methods}

Forty-nine delegates (male $N=27$, female $N=22$ ) were selected and assessed throughout the course. The selection criteria were based on CV parameters including previous oral or poster presentations and publications. The whole process ran through the online portal (http://esmsc.gr/), and the applicants were also asked to write a small statement explaining the reasons for their application, in order to assess their personal interest in surgical specialties. The mean age was 23.7 years $(\min =20, \max =30$, and $\mathrm{SD}=2.47)$. Out of these, $26.5 \%(N=13)$ were students from KCL at Year 3 and the remaining $73.5 \%(N=36)$ from Greek Universities, from which $32.7 \%$ were Year $4(N=16), 22.4 \%$ Year $5(N=11)$, and $18.4 \%$ were Year $6(N=9)$ (Figure 1$)$. KCL Year 3 is the first clinical rotation of the students and the experience is equal to 4th and 5th Year in Greek Universities. None of the medical students had any previous experience in wet lab courses or the operating room, and therefore their relevant experience was assumed to be similar.

The course manual (2nd Edition) was designed to cover all the background knowledge that is required for the consolidation of the course curriculum, and it was given to the students, on their arrival.

Precourse MCQ exam was performed on participants' arrival, and the same exam was conducted on completion of the course. This MCQ reflects on the course curriculum and consists of 32 questions of average difficulty. Participants were assessed before and after every wet lab skills' module, with DOPS assessments, by qualified trainers from the UK and Greece. All DOPS forms were validated by the ISCP (Table 1), simplified and standardized for the purposes of the course. We used 3 different markings, $\mathrm{N}$ (or 0 ) for not able to perform 
TABLE 1: An example based on the DOPS form of Intercollegiate Surgical Curriculum Programme (ISCP).

(a)

\begin{tabular}{ll}
\hline \multirow{2}{*}{ Domain } & Rating \\
& N/D/S \\
\hline
\end{tabular}

(1) Describes indications, anatomy, procedure, and complications to assessor

(2) Obtains consent, after explaining procedure and possible complications to patient

(3) Prepares for procedure according to an agreed protocol

(4) Administers effective analgesia or safe sedation (if no anaesthetist)

(5) Demonstrates good asepsis and safe use of instruments and sharps

(6) Performs the technical aspects in line with the guidance notes

(7) Deals with any unexpected event or seeks help when appropriate

(8) Completes required documentation (written or dictated)

(9) Communicates clearly with patient and staff throughout the procedure

(10) Demonstrates professional behaviour throughout the procedure

(b)

\begin{tabular}{|c|c|}
\hline Level 0 & $\begin{array}{l}\text { Insufficient evidence observed to support a summary } \\
\text { judgement }\end{array}$ \\
\hline Level 1 & Unable to perform the procedure under supervision \\
\hline & Able to perform the procedure under supervision \\
\hline Level 3 & $\begin{array}{l}\text { Able to perform the procedure with minimum } \\
\text { supervision (needed occasional help) }\end{array}$ \\
\hline Level 4 & $\begin{array}{l}\text { Competent to perform the procedure unsupervised } \\
\text { (and could deal with any complications that arose) }\end{array}$ \\
\hline
\end{tabular}

(or not observed), D (or 1) for development needed, and S (or 2) for satisfactory. This reflects on specific parts of each wet lab module. Global marking on scale $0-4$ was used to assess the overall competence of the delegates to perform each module independently or with assistance.

On the suturing station, participants were assessed on the ability to perform independently interrupted, subcuticular, and mattress sutures (Figure 5). During the Wound Debridement module, delegates were assessed on their ability to clean a wound and suture it primarily. Using certain questions, they were also prompted on their understanding of the process. ORIF station delegates were expected to perform the relevant skill on small plastic pieces, using the relevant equipment (Figure 2). The IV Seldinger technique was conducted (Figure 3) on swine skin flaps, using urethral catheters and the relevant equipment. Participants were tested on their ability to understand the indications and the complications and independently perform the procedure.

With regard to the in vivo experiments, local standard operational procedure (SOP) protocol for anaesthesia and

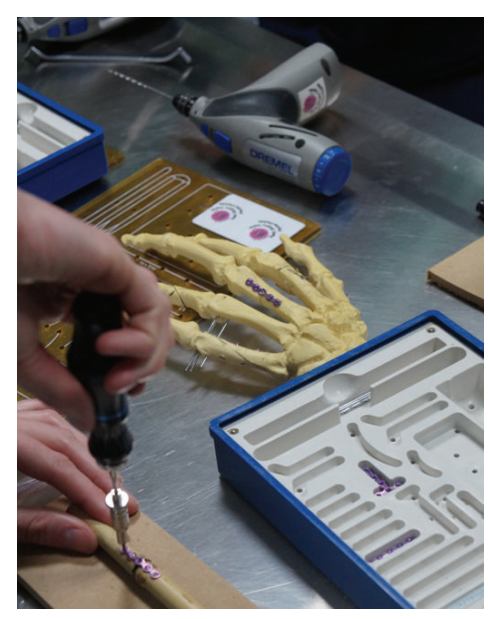

FIGURE 2: Open Reduction Internal Fixation of hand fractures.

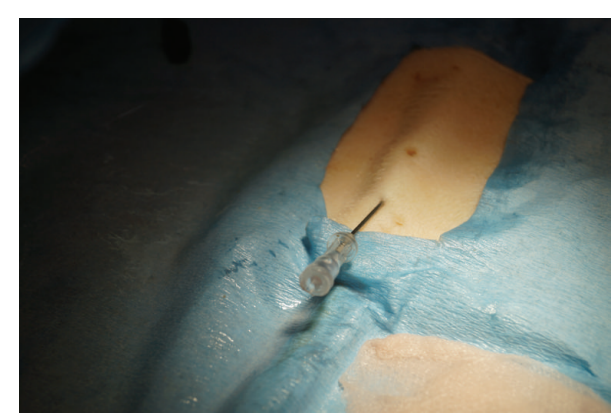

FIGURE 3: IV Seldinger technique using pig skin flaps.

preparation of the pig was used accordingly, whilst on the first in vivo experiment participants were actively assisting on anatomy demonstrations, as well as chest tube insertion. They were tested on their ability to perform abdominal wall dissection through layers independently and closure. DOPS assessments were used on the same basis as before. During the second in vivo experiment, delegates had the opportunity to assist a senior surgeon preparing the pig for heart transplant (Figure 4). During this module, we used only the global rating scale (0-4), as it was above the level of expected skills.

Detailed feedback forms were handled to the delegates on their arrival. A global rating scale of 1-10 was used for every course lecture or skills module. Overall satisfaction questions were included on it in order to acquire an idea of the overall setting of the course.

Statistical analysis of the MCQ and DOPS results was conducted using paired t-test associations before and after each module. Independent $t$-test associations were performed to assess the difference in the performance between different groups of participants, that is, Years 3 and 4 versus Years 5 and 6 students, as well as UK versus Greek students. For this purpose, we used IBM SPSS for Mac (Edition 22, Armonk, NY: IBM Corp.). $P$ values less than .05 were thought to be statistically significant. 
TABLE 2: Delegates mean DOPS scores $(\mathrm{N}=0 / \mathrm{D}=1 / \mathrm{S}=2)$ and global rating scale $(0-4)$.

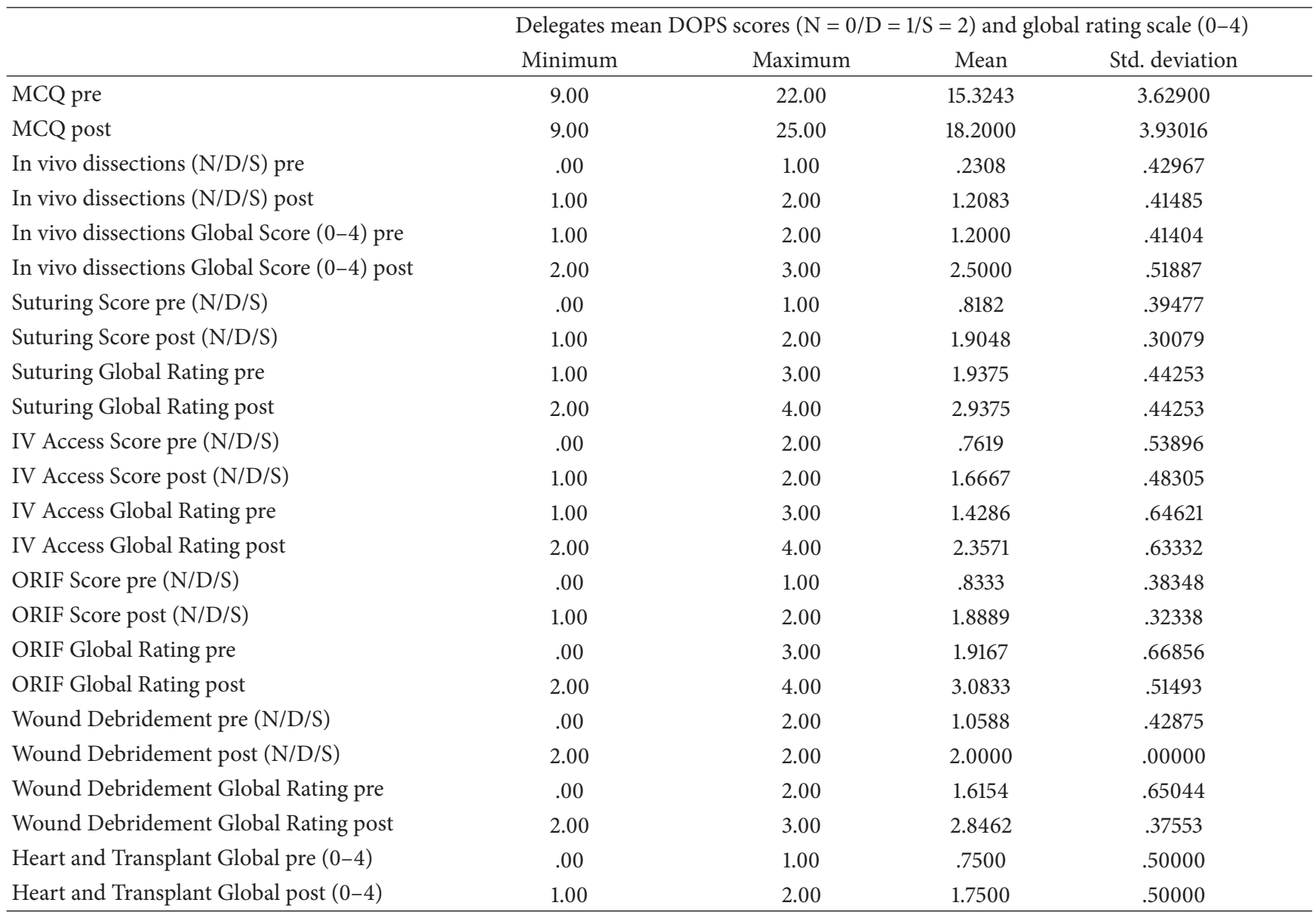

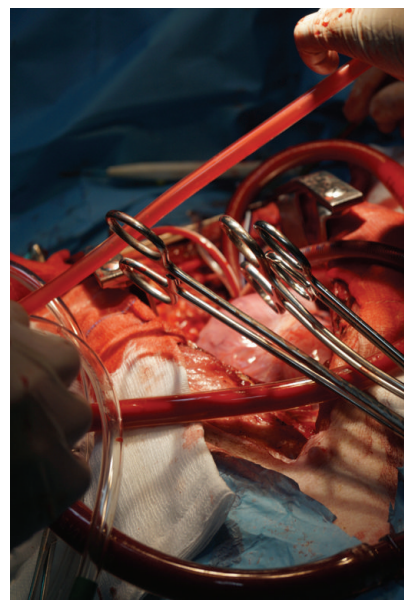

FIgURE 4: Heart and Transplant In Vivo Module on swine model.

\section{Results}

Mean MCQ score of the delegates before the course was $15.32 / 32(\min =9, \max =22$, and $\mathrm{SD}=3.63)$ versus $18.2 / 32$ $(\min =9, \max =25$, and $\mathrm{SD}=3.93)$ after course. The mean difference was $2.67(\min =1.51, \max =3.81$, and $\mathrm{SD}=3.23$,

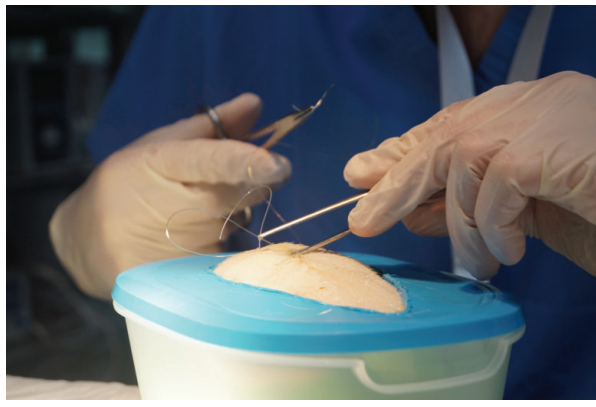

FIGURE 5: Suturing station on pig skin flaps.

$P<0.005)$. With regard to wet lab skills' module, the mean score of in vivo dissections (Skill 1) before teaching was 0.23 out of $2(\min =0, \max =1$, and $\mathrm{SD}=0.43)$ with a mean global rating (scale $0-4)$ of $1.2(\min =1, \max =2$, and $\mathrm{SD}=0.41)$, versus postteaching mean $=1.21$ out of $2(\min =1, \max =2$, and $\mathrm{SD}=0.41)$ and mean global rating of $2.5(\mathrm{~min}=2$, $\max$ $=3$, and $\mathrm{SD}=0.51$, score $0-4)$. The mean difference before and after in vivo dissections teaching was 0.96 out of 2 ( $\mathrm{min}$ $=0.80, \max =1.10$, and $\mathrm{SD}=0.36, P<0.005)$ and the mean global rating improvement was $1.29(\mathrm{~min}=0.93, \max =1.63$, and $\mathrm{SD}=0.61, P<0.005)$ (Tables 2 and 3$)$. 


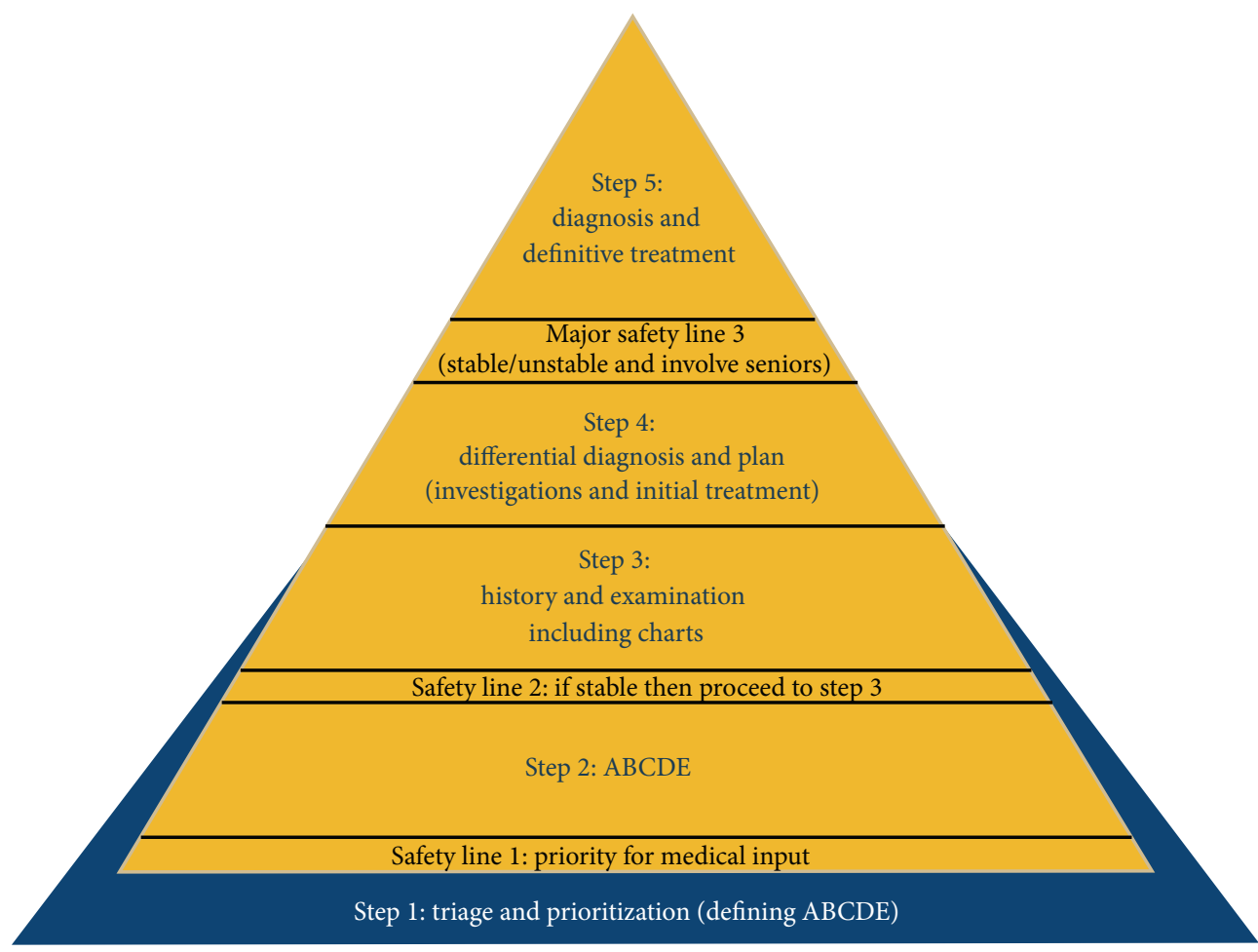

FIgURE 6: Safety triangle as published on the relevant manual (Sideris, Papalois et al.).

TABLE 3: Comparison of the mean difference between MCQ (pre- and postcourse) and DOPS (pre- and postmodule).

\begin{tabular}{|c|c|c|c|c|c|c|c|}
\hline \multirow{3}{*}{ Module } & \multicolumn{7}{|c|}{ Comparison of MCQ and DOPS scores } \\
\hline & \multicolumn{3}{|c|}{ Paired differences } & \multicolumn{2}{|c|}{$95 \%$ confidence interval of the difference } & \multirow{2}{*}{$t$-test } & \multirow{2}{*}{ Sig. } \\
\hline & Mean & $\mathrm{SD}$ & SE mean & Lower & Upper & & \\
\hline MCQ post-MCQ pre & 2.66667 & 3.23715 & .56352 & 1.51882 & 3.81451 & 4.732 & .000 \\
\hline In vivo dissections $(\mathrm{N} / \mathrm{D} / \mathrm{S})$ & .95833 & .35864 & .07321 & .80689 & 1.10977 & 13.091 & .000 \\
\hline In vivo dissections global $(0-4)$ & 1.28571 & .61125 & .16336 & .93279 & 1.63864 & 7.870 & .000 \\
\hline Suturing Score $(\mathrm{N} / \mathrm{D} / \mathrm{S})$ & 1.09524 & .30079 & .06564 & .95832 & 1.23216 & 16.686 & .000 \\
\hline Suturing Global (0-4) & 1.00000 & .51640 & .12910 & .72483 & 1.27517 & 7.746 & .000 \\
\hline IV access $(\mathrm{N} / \mathrm{D} / \mathrm{S})$ & .90476 & .30079 & .06564 & .76784 & 1.04168 & 13.784 & .000 \\
\hline IV access global (0-4) & .92857 & .26726 & .07143 & .77426 & 1.08288 & 13.000 & .000 \\
\hline ORIF (N/D/S) & 1.05556 & .41618 & .09809 & .84860 & 1.26252 & 10.761 & .000 \\
\hline ORIF Global (0-4) & 1.16667 & .38925 & .11237 & .91935 & 1.41398 & 10.383 & .000 \\
\hline Wound Debridement (N/D/S) & .93750 & .44253 & .11063 & .70169 & 1.17331 & 8.474 & .000 \\
\hline Wound Debridement Global (0-4) & 1.23077 & .59914 & .16617 & .86871 & 1.59283 & 7.407 & .000 \\
\hline Heart and Transplant Global Score $(0-4)$ & 1.10000 & .31623 & .10000 & .87378 & 1.32622 & 11.000 & .000 \\
\hline
\end{tabular}

With regard to ex vivo suturing station (Skill 2), the mean score before teaching was 0.82 out of $2(\min =0, \max =1$, and $\mathrm{SD}=0.39)$ with a global rating of $1.94(\min =1, \max =3$, and $\mathrm{SD}=0.44$, scale $0-4)$. The mean score after teaching was 1.90 out of $2(\min =1, \max =2$, and $S D=0.30)$ and the mean global rating was $2.94(\min =2, \max =4$, and $\mathrm{SD}=0.44)$. The mean difference before and after teaching was 1.09 ( $\mathrm{min}$ $=0.96, \max =1.23$, and $\mathrm{SD}=0.30, P<0.005)$ with a global rating improvement of $1.00(\min =0.72, \max =1.27$, and SD $=0.52, P<0.005)$ (Tables 2 and 3$)$.

Delegates mean score in the IV Access Ex Vivo station (Skill 3$)$ before teaching was 0.76 out of $2(\min =0, \max =2)$ with mean global rating of $1.42(\min =1, \max =3$, and $\mathrm{SD}=$ 0.64 , scale $0-4)$. The mean score after teaching was 1.66 out of $2(\min =1, \max =2$, and $\mathrm{SD}=0.48)$ with a mean global rating of $2.35(\min =2, \max =4$, and $\mathrm{SD}=0.63$, scale $0-4)$. 
The mean difference in the score was $0.90(\min =0.76$, $\max$ $=1.04$, and $\mathrm{SD}=0.30, P<0.005)$ and in the global rating was $0.92(\min =0.77, \max =1.08$, and $\mathrm{SD}=0.26)($ Tables 2 and 3$)$.

The mean score in the ORIF ex vivo station (Skill 4) before teaching was 0.83 out of $2(\min =0, \max =1$, and $\mathrm{SD}=0.38)$ and the mean global rating was $1.91(\min =0, \max =3$, and SD $=0.66$, scale $0-4)$. The mean score after teaching was 1.88 out of $2(\min =1, \max =2$, and SD $=0.32)$ and the mean global rating was $3.08(\min =2, \max =4$, and $\mathrm{SD}=0.51)$. The mean difference in the score was $1.05(\min =0.85, \max =1.26$, and $\mathrm{SD}=0.41, P<0.005)$ and in the global rating was $1.16(\mathrm{~min}=$ $0.91, \max =1.41$, and $\mathrm{SD}=0.39, P<0.005)$ (Tables 2 and 3 ).

In the Wound Debridement ex vivo module (Skill 5), the mean score before teaching was 1.05 out of $2(\min =0$, $\max$ $=2$, and $\mathrm{SD}=0.42)$ and the mean global rating was 1.61 ( $\mathrm{min}$ $=0, \max =2$, and $\mathrm{SD}=0.65$, scale $0-4)$. The mean score after teaching was 2.00 out of $2(\min =2, \max =3$, and $S D=0.00)$ and the mean global rating was $2.84(\min =2, \max =3$, and $\mathrm{SD}=0.38$, scale $0-4)$. The mean difference in the score was $0.93(\min =0.7, \max =1.17, P<0.005)$ and in the Global Score was $1.23(\min =0.87, \max =1.59$, and $\mathrm{SD}=0.59, P<0.005)$.

Global rating of the delegates for the Heart and Transplant In vivo Module (Skill 6) before teaching was $0.75(\mathrm{~min}=0$, $\max =1$, and $\mathrm{SD}=0.50$, scale $0-4$ ) versus mean global rating of $1.75(\min =1, \max =2$, and $\mathrm{SD}=0.5$, scale $0-4)$. The mean difference was $1.10(\min =0.83, \max =1.32$, and $\mathrm{SD}=0.31$, $P<0.005$ ) (Tables 2 and 3).

With regard to independent $t$-test associations, the mean MCQ score after the course was 15.92 for UK students (Year 3) versus 19.29 for Greek Students (Year 4-6). There was a statistically significant better performance of Greek Students in the mean MCQ score after the course $(P=0.09, P=0.05)$. The mean score in the in vivo dissections before and after completion of module (Skill 1) was .00 and 1.00 for the UK (Year 3) students versus 0.38 and 1.36 for (Year 4-6) Greek students. Therefore, there was an improved performance of Greek Students in the in vivo dissections' score before $(P=0.027, P=0.09)$ and after the completion of module $(P=0.034, P=0.019)$. There was no other statistical significant difference in any comparison between UK and Greek students.

Concerning the comparison between the 2 groups of Year 3 and Year 4 students versus Year 5 and Year 6 students, there was a statistically significantly better performance of the group of Year 5 and Year 6 students in the mean MCQ score after completion of course (mean score 20.37 versus 16.77, $P=0.034$ and $P=0.014$ ) as well as in the mean score before starting the in vivo dissections' module (Skill 1$)$ ( 0.55 versus $0.06, P=0.03$ and $P=0.023$ ). There was no other statistically significant difference in the performance of those 2 groups.

The mean overall feedback from the participants was 8.78 out of $10(\min =7.72, \max =9.87$, and $\mathrm{SD}=0.56)$. There are those who think that ESMSC should be provided by the Medical School $(9.86$ out of $10, \min =8, \max =10$, and SD $=$ $0.43)$. Also, the overall idea was ranked with 9.42 out of 10 $(\min =8, \max =10$, and SD $=0.71)$ and the general concept of the curriculum was given $9.34 / 10(\min =7, \max =10$, and SD $=0.82$ ). On direct questioning, the delegates think that this course helps in developing the principles of surgical approach
(9.5 out of $10, \min =7, \max =10$, and $\mathrm{SD}=0.86)$ and the curriculum was given $9.30 / 10$ in terms of how useful this may be for their future surgical career $(\min =6, \max =10$, and $\mathrm{SD}=0.99)$. Most of the attendees seem to be interested in a surgical career $($ mean $=8.18$, mean $=3, \max =10$, and $\mathrm{SD}=$ 1.97). Detailed feedback is summarized in Table 4 .

\section{Discussion}

Quality of surgical training is one of the biggest challenges that reflect directly onto patients' safety. There have been multiple models which aim to teach either basic $[7,8]$ or advanced skills [1, 2, 4-6] throughout the surgical journey. Surgical training is changing throughout Europe as the result of new legislation for working hours. Increasing workload along with the European Working Time Directive (EWTD) could potentially result in shorter surgical specialty training, which many have an impact on the adequacy of the training years in specialty [9]. This creates an argument for starting surgery-orientated training and skills-based training while still in Medical School.

On the other hand, increasing financial pressure is an additional challenge that has to be dealt with $[10,11]$. Recently, bench model-based teaching of clinical skills has gained wide acceptance, though the outcomes still depend on the quality of facilities and trainers [10]. The need of getting trainees exposed to a wider variety of surgical skills before assisting in the theatre room is all the more clear given the increasing workload. Practicing skills in theatre could even double up the operating time [11] and even in some cases compromise patients' safety.

What is more, there has been a revolution of new surgical technologies in the last 15 years, such as laparoscopic surgery, robotic surgery, surgical endoscopy, and Natural Orifice Endoluminal Surgery. All those technologies have a steep learning curve and require long hands-on training. All surgical training tends to increasingly involve simulation training, which has been copied from the airline industry. This way of training is likely to develop into the major pattern of training for the current medical students.

There is no doubt that developing simulation models on which trainees can practice their skills would be beneficial for their long-term acquisition of required competences, in order to become safe and efficient surgeons $[12,13]$. The main question though remains at which stage the right time to introduce future surgeons in those courses is. Moreover, it is vital to think about the right choice of each course curriculum, with specific aims and targets $[14,15]$, and subsequently to establish an objective validation model, which would prove that the outcome is worth money, time, and effort. There have been multiple validation methods [13], and in our case we used the Workplace Based Assessments (WPBA), and specifically the DOPS forms, from the ISCP $[14,16]$.

Introducing medical students to ex vivo model has been successful throughout a relatively extensive period of time $[7,8]$. This remains a cost-effective option, given the fact that ex vivo modules are cheaper compared to when you attempt to include in vivo swine model, in order to achieve the highest fidelity model. Students seem to respond favourably 
TABLE 4: Overall feedback analysis (scale $0-10,0=$ disagree/worst, $10=$ completely agree/best).

\begin{tabular}{|c|c|c|c|c|c|c|c|}
\hline & \multicolumn{7}{|c|}{ Analysis of overall feedback (ascending values) } \\
\hline & \multirow{2}{*}{$\begin{array}{l}\text { Range } \\
\text { Statistic }\end{array}$} & \multirow{2}{*}{$\begin{array}{c}\text { Minimum } \\
\text { Statistic }\end{array}$} & \multirow{2}{*}{$\begin{array}{c}\text { Maximum } \\
\text { Statistic }\end{array}$} & \multicolumn{2}{|c|}{ Mean } & \multirow{2}{*}{$\begin{array}{c}\text { Std. Deviation } \\
\text { Statistic }\end{array}$} & \multirow{2}{*}{$\begin{array}{l}\text { Variance } \\
\text { Statistic }\end{array}$} \\
\hline & & & & Statistic & Std. error & & \\
\hline ECG Workshop & 7.00 & 3.00 & 10.00 & 7.7187 & .29524 & 1.67012 & 2.789 \\
\hline Food & 10.00 & .00 & 10.00 & 7.7333 & .42327 & 2.31834 & 5.375 \\
\hline Surgical Oncology (IL) & 4.00 & 5.00 & 9.00 & 7.7667 & .27411 & 1.50134 & 2.254 \\
\hline Website & 6.00 & 4.00 & 10.00 & 7.7931 & .33432 & 1.80038 & 3.241 \\
\hline Breaks & 10.00 & .00 & 10.00 & 7.8000 & .51950 & 2.84544 & 8.097 \\
\hline Rectal Cancer (IL) & 8.00 & 2.00 & 10.00 & 7.8750 & .47895 & 2.34637 & 5.505 \\
\hline ENT CbL & 7.00 & 3.00 & 10.00 & 7.9355 & .34992 & 1.94826 & 3.796 \\
\hline Heart and Transplant In Vivo Module (Sk6) & 7.00 & 3.00 & 10.00 & 7.9667 & .39966 & 2.18905 & 4.792 \\
\hline Vascular CbL & 6.00 & 4.00 & 10.00 & 7.9677 & .32622 & 1.81629 & 3.299 \\
\hline Interest in Surgical Career & 7.00 & 3.00 & 10.00 & 8.1875 & .34907 & 1.97464 & 3.899 \\
\hline Cardiothoracic CbL & 8.00 & 2.00 & 10.00 & 8.2813 & .30531 & 1.72710 & 2.983 \\
\hline Fluids and Analgesia Workshop & 6.00 & 4.00 & 10.00 & 8.4687 & .27307 & 1.54470 & 2.386 \\
\hline Basic Science Workshops Overall & 6.00 & 4.00 & 10.00 & 8.6774 & .32918 & 1.83280 & 3.359 \\
\hline DKA/ACS/Sepsis CbL & 4.00 & 6.00 & 10.00 & 8.7097 & .16844 & .93785 & .880 \\
\hline Research in Medical Education (IN) & 5.00 & 5.00 & 10.00 & 8.7187 & .26272 & 1.48616 & 2.209 \\
\hline In vivo dissections (Sk1) & 5.00 & 5.00 & 10.00 & 8.7500 & .24593 & 1.39122 & 1.935 \\
\hline Orthopaedics CbL & 7.00 & 3.00 & 10.00 & 8.7742 & .32696 & 1.82043 & 3.314 \\
\hline Statistic Mean Feedback & 2.15 & 7.72 & 9.87 & 8.7882 & .08485 & .56284 & .317 \\
\hline Advances in Valve Surgery (IL) & 5.00 & 5.00 & 10.00 & 8.8333 & .29588 & 1.62063 & 2.626 \\
\hline GS and HPB CbL & 4.00 & 6.00 & 10.00 & 8.8437 & .18022 & 1.01947 & 1.039 \\
\hline Wound Debridement - Ex Vivo (Sk5) & 6.00 & 4.00 & 10.00 & 8.8710 & .26938 & 1.49982 & 2.249 \\
\hline Abdominal Trauma CbL & 5.00 & 5.00 & 10.00 & 8.8710 & .26535 & 1.47743 & 2.183 \\
\hline Consent CbL & 7.00 & 3.00 & 10.00 & 8.8710 & .23981 & 1.33521 & 1.783 \\
\hline Burns CbL & 5.00 & 5.00 & 10.00 & 8.9063 & .23053 & 1.30407 & 1.701 \\
\hline IV Access - Ex Vivo (Sk3) & 3.00 & 7.00 & 10.00 & 8.9375 & .19540 & 1.10534 & 1.222 \\
\hline Shock Workshop & 3.00 & 7.00 & 10.00 & 8.9375 & .19017 & 1.07576 & 1.157 \\
\hline Suturing Ex Vivo (Sk2) & 7.00 & 3.00 & 10.00 & 8.9687 & .27121 & 1.53422 & 2.354 \\
\hline Chapters Selection in Manual & 3.00 & 7.00 & 10.00 & 9.0000 & .15554 & .87988 & .774 \\
\hline Appearance of the Manual & 4.00 & 6.00 & 10.00 & 9.0000 & .17961 & 1.01600 & 1.032 \\
\hline ESMSC "safety triangle" & 5.00 & 5.00 & 10.00 & 9.0000 & .23187 & 1.29099 & 1.667 \\
\hline Lecture Surgical Approach (IN) & 4.00 & 6.00 & 10.00 & 9.0000 & .20080 & 1.13592 & 1.290 \\
\hline Support during Course & 5.00 & 5.00 & 10.00 & 9.0333 & .26472 & 1.44993 & 2.102 \\
\hline Was it a useful course (Day 1) & 3.00 & 7.00 & 10.00 & 9.0625 & .17925 & 1.01401 & 1.028 \\
\hline Faculty Selection & 3.00 & 7.00 & 10.00 & 9.1000 & .19387 & 1.06188 & 1.128 \\
\hline ORIF- Ex Vivo & 7.00 & 3.00 & 10.00 & 9.1290 & .27334 & 1.52188 & 2.316 \\
\hline Course Lead & 9.00 & 1.00 & 10.00 & 9.1724 & .32570 & 1.75395 & 3.076 \\
\hline ABG Workshop & 4.00 & 6.00 & 10.00 & 9.2813 & .18640 & 1.05446 & 1.112 \\
\hline Was it a useful course (Day 2) & 4.00 & 6.00 & 10.00 & 9.3000 & .18036 & .98786 & .976 \\
\hline General Concept Day 1 & 5.00 & 5.00 & 10.00 & 9.3000 & .20982 & 1.14921 & 1.321 \\
\hline General Concept Day 2 & 3.00 & 7.00 & 10.00 & 9.3438 & .14625 & .82733 & .684 \\
\hline Hand Emergencies CbL & 3.00 & 7.00 & 10.00 & 9.3548 & .16427 & .91464 & .837 \\
\hline Surgical Approach Ability - Day 1 & 3.00 & 7.00 & 10.00 & 9.3750 & .14722 & .83280 & .694 \\
\hline Overall Rate of the Idea & 2.00 & 8.00 & 10.00 & 9.4194 & .12930 & .71992 & .518 \\
\hline Facilities & 3.00 & 7.00 & 10.00 & 9.4667 & .14169 & .77608 & .602 \\
\hline
\end{tabular}


TABle 4: Continued.

\begin{tabular}{|c|c|c|c|c|c|c|c|}
\hline & \multicolumn{7}{|c|}{ Analysis of overall feedback (ascending values) } \\
\hline & \multirow{2}{*}{$\begin{array}{l}\text { Range } \\
\text { Statistic }\end{array}$} & \multirow{2}{*}{$\begin{array}{l}\text { Minimum } \\
\text { Statistic }\end{array}$} & \multirow{2}{*}{$\begin{array}{l}\text { Maximum } \\
\text { Statistic }\end{array}$} & \multicolumn{2}{|c|}{ Mean } & \multirow{2}{*}{$\begin{array}{l}\text { Std. Deviation } \\
\text { Statistic }\end{array}$} & \multirow{2}{*}{$\begin{array}{l}\text { Variance } \\
\text { Statistic }\end{array}$} \\
\hline & & & & Statistic & Std. error & & \\
\hline Surgical Approach Ability Day 2 & 3.00 & 7.00 & 10.00 & 9.5000 & .15720 & .86103 & .741 \\
\hline $\begin{array}{l}\text { Should Medical School Provide It? } \\
\text { (Feedback from Day } 1 \text { ) }\end{array}$ & 3.00 & 7.00 & 10.00 & 9.7813 & .10752 & .60824 & .370 \\
\hline $\begin{array}{l}\text { Should Medical School Provide It? } \\
\text { (Feedback from Day 2) }\end{array}$ & 2.00 & 8.00 & 10.00 & 9.8667 & .07927 & .43417 & .189 \\
\hline
\end{tabular}

$\mathrm{CbL}=$ case-based lecture; $\mathrm{IL}$ = invited lecture; $\mathrm{IN}$ = introductory lecture; $\mathrm{Sk}=$ in vivo/ex vivo modules.

to these initiatives, and their objective assessments show improvement in terms of the basic surgical skills [7].

In our case, we attempted to establish a combined course, which aims to help students consolidate the basic surgical knowledge, establish the principles of the surgical approach, and practice on the basic hands-on skills. The choice of modules (Table 2) was mainly based on the essence to cover from alpha to omega all the relevant experience that a medical student would need, in order to be safe and efficient in both theatre and surgical wards. The introduction of in vivo modules would be the ideal high-fidelity simulation environment, where medical students will be exposed to all theatre equipment and will perform their first manipulations with real tissue. This would build up students' confidence and safety, before they actually assist in theatre. The whole idea was to design a step-by-step approach, so that delegates would be able to take their skills forward to the next step. With regard to the in vivo transplant module, despite the limitations in the actual students' input, it still has a mentorship value as it is a unique opportunity to interact with the surgeon. Also the students are able to identify and consolidate step by step the thoracic anatomy as well as the cardiopulmonary physiology principles and understand the outlines of Transplant Surgery.

The question still remains about the cost effectiveness of the in vivo model in the undergraduate surgical education. Despite high cost being an obstacle, in vivo dissections were deemed really valuable in the feedback (Table 4). Furthermore, from the actual objective DOPS scores, we could assume that this was reflected in the mean difference in the scores before and after the in vivo modules (basic dissections and Heart and Transplant Surgery), which were statistically significantly improved $(P<0.005)$. Despite the limitations in the extent and detail of those assessments, we could support though that there is a benefit in the performance of the delegates.

MCQ exams, before and after completion of the course, were used to validate the quality of knowledge acquired from the basic science and case-based learning cores. The mean improvement was 2.33/32 $(P<0.005)$, and therefore this seems to work effectively. The MCQ exam is mainly designed to cover basic aspects of the taught lectures. In terms of the hands-on skills, we used DOPS forms to validate objectively the level of acquired competence. In every case, the scores were improved significantly after completion of each module $(P<0.005)$. For this purpose, we followed
Miller's Pyramid Principles along with WPBA to validate it [16]. This follows the fundamental assumption that a young trainee (or a medical student in our case) would not be able to perform a skill completely independently; however, it still helps towards the acquisition of a higher level of competence on a specific skill on the scale 0-4. Finally, consolidation of knowledge is attempted via distribution of the international course manual, which aims to cover all the aspects of the course. We decided to distribute the manual on the participants' arrival, in order to achieve a similar background knowledge for every participant and hence to evaluate the effectiveness of the course itself. In any other case, it would have been more complicated to achieve a homogenous sample of students.

With regard to the DOPS scores, there has been an improvement in all modules before and after completion $(P<0.05)$. This was clear in both the objective comparison DOPS (Table 3), as well as in students' feedback (Table 4), suggesting that our course may be an effective teaching model. Also, the vast majority of scores suggest that students lack more in hands-on experience rather than anything else. There is significant improvement even in simple modules, like suturing.

Comparing British versus Greek students, we have to take into consideration that all British students are Year 3 students, whereas Greek students come from a mixture of Years 4-6. That may justify the slightly better performance of Greek students in the 2nd MCQ exam, as well as being more familiar with basic dissections (Skill 1), with no further significance. A more interesting comparison lies between the group consisting of Year 3 students in the UK and Year 4 students in Greece versus Years 5 and 6. Year 3 is the first clinical year in the UK, whilst Year 4 is the equivalent for Greek students. Again, on that occasion there are minor differences in the performance during the final MCQ exam as well as in in vivo module 1 . This supports the gross homogeneity of the groups, as there are minor differences in the vast majority of the modules. Also, it underlines the lack of hands-on exposure, as final year students have similar performance with Year 3 and Year 4 students.

Finally, a last point worth commenting on is the selection of students and faculty from various institutions around Greece and the UK. There remains significant variation in surgical training amongst EU countries, despite all attempts to establish uniform qualifications, such as the European Boards. Therefore, it will be increasingly necessary to enhance 
the communication and interactions between medical students from diverse training systems to identify improvements and exchange ideas and views on training challenges. For that reason, it is very interesting to organize a joint course for British and Greek medical students.

From the medical students' point of view, they seemed to appreciate the acquired knowledge, which is directly reflected by their feedback (Table 4). The average overall feedback was $8.78 / 10(7.72-9.87, \mathrm{SD}=0.56)$, and the students believe that this would be implemented on the Medical School Curriculum for the acquisition of the relevant competences $(9.86 / 10,8-10$, and SD $=0.43)$. Also, they felt very strongly about having more confidence in approaching a surgical patient $(9.50 / 10,7-10$, and $\mathrm{SD}=0.86)$, which was improved from day 1 to day 2 (day 1 confidence 9.37/10, 7-10, and $\mathrm{SD}=0.83)$. They also felt that they were well-supported throughout the course $(9.03 / 10,5-10$, and SD = 1.44), which is also reflected in the good combination of faculty from the UK and Greece $(9.10 / 10,7-10$, and SD = 1.06).

\section{Conclusions}

Wet lab simulation-based combined courses seem to be effective in the acquisition of theoretical knowledge, as well as hands-on skills for medical students. Combination of ex vivo and in vivo skills could potentially offer a step further in the preparation of the medical students, who would be interested to pursue a career in surgery. The question still remains as to what the right balance of time and money invested towards the most cost-effective model for this purpose is.

\section{Ethical Approval}

This study was performed according to European and National Legislation, Directive 63/2010, PD 56/ April 2013. Reference Number of the License is Michail Ch. Sideris and Apostolos Papalois 7095/05-11-2014 (revised 884 28/4/2015).

\section{Conflict of Interests}

The authors declare no conflict of interests.

\section{Authors' Contribution}

Michail Sideris and Apostolos Papalois are equal contributors/authors, leaders of the project, designers of the course curriculum, and main editors of the manual. Georgios Tsoulfas is a member of the faculty of the course and took the lead for the in vivo dissections (anatomy demonstration of the abdomen). Sanjib Majumder is a member of the faculty of the course and took the lead for suturing and Open Reduction Internal Fixation ex vivo modules. Konstantinos Toutouzas is a member of the faculty of the course and participated in the in vivo dissections (abdominal anatomy). Efstratios Koletsis is a member of the faculty of the course and led the in vivo dissections of the thorax (cardiothoracic modules). Panagiotis Dedeilias is a member of the faculty of the course and took the lead for Heart and Transplant In Vivo Module. Nikolaos
Lymperopoulos is a member of the faculty of the course and an assistant to Mr. S. Majumder for the ORIF and Suturing Ex Vivo station. Savvas Papagrigoriadis is a senior advisor of the course curriculum and manual and international lecturer during course. Vassilios Papalois is a senior advisor of the course curriculum and manual. Georgios Zografos is a senior advisor of the course curriculum and manual and took the lead for the scientific committee of the course. All the authors have contributed to data collection, and they have been formal assessors as well in the relevant modules (Workplace Based Assessments of the Medical Students).

\section{Acknowledgments}

Special thanks are due to Experimental Research Centre of ELPEN Faculty. The authors would like to thank all the staff of the E.R.C. ELPEN and especially Georgios Stagias, Felasa C, and Nikolaos Psychalakis, Felasa C, and the Medical Students' Committee, Nikolaos Staikoglou, Efstratia Georgopoulou, Ismini Tsagkaraki, Georgios Paparoidamis, Maria Toumpourleka, and Stefanos Karamaroudis. Essential Skills in the Management of the Surgical Cases (ESMSC) is funded by the Experimental Research Centre ELPEN S.A., and it is provided for free to medical students around Europe.

\section{References}

[1] Y. W. Ng and Y. F. Fong, "Get 'real' with hysteroscopy using the pig bladder: a 'uterine' model for hysteroscopy training," Annals of the Academy of Medicine, vol. 42, no. 1, pp. 18-23, 2013.

[2] M. Golriz, M. Hafezi, C. Garoussi et al., "Do we need animal hands-on courses for transplantation surgery?" Clinical Transplantation, vol. 27, supplement 25, pp. 6-15, 2013.

[3] S. K. Narayanan, R. C. Cohen, and A. Shun, "Technical tips and advancements in pediatric minimally invasive surgical training on porcine based simulations," Pediatric Surgery International, vol. 30, no. 6, pp. 655-661, 2014.

[4] J. Rosenberg, I. Presch, H. C. Pommergaard, J. Burcharth, and M. Bagot D'Arc, "An animal model to train Lichtenstein inguinal hernia repair," Hernia, vol. 17, no. 2, pp. 255-258, 2013.

[5] M. La Torre and C. Caruso, "The animal model in advanced laparoscopy resident training," Surgical Laparoscopy, Endoscopy and Percutaneous Techniques, vol. 23, no. 3, pp. 271-275, 2013.

[6] J. Martinek, S. Suchanek, M. Stefanova et al., “Training on an ex vivo animal model improves endoscopic skills: a randomized, single-blind study," Gastrointestinal Endoscopy, vol. 74, no. 2, pp. 367-373, 2011.

[7] F. Bauer, N. Rommel, K. Kreutzer et al., "A novel approach to teaching surgical skills to medical students using an ex vivo animal training model," Journal of Surgical Education, vol. 71, no. 4, pp. 459-465, 2014.

[8] R. Denadai, M. Oshiiwa, and R. Saad-Hossne, "Teaching elliptical excision skills to novice medical students: a randomized controlled study comparing low- and high-fidelity bench models," Indian Journal of Dermatology, vol. 59, no. 2, pp. 169-175, 2014.

[9] L. Depypere, L. De Jonghe, W. Peetermans, and P. De Leyn, "Does the implementation of European working time directive (EWTD) have an effect on surgical training in a Flemish 
Teaching Hospital network?” Acta Chirurgica Belgica, vol. 114, no. 5, pp. 299-303, 2014.

[10] A. Dubrowski and H. MacRae, "Randomised, controlled study investigating the optimal instructor: student ratios for teaching suturing skills," Medical Education, vol. 40, no. 1, pp. 59-63, 2006.

[11] M. Bridges and D. L. Diamond, "The financial impact of teaching surgical residents in the operating room," American Journal of Surgery, vol. 177, no. 1, pp. 28-32, 1999.

[12] K. A. Barsness, "Trends in technical and team simulations: challenging the status Quo of surgical training," Seminars in Pediatric Surgery, vol. 24, no. 3, pp. 130-133, 2015.

[13] N. G. Patil, H. Saing, and J. Wong, "Role of OSCE in evaluation of practical skills," Medical Teacher, vol. 25, no. 3, pp. 271-272, 2003.

[14] A. W. Phillips and A. Madhavan, "A critical evaluation of the intercollegiate surgical curriculum and comparison with its predecessor the 'Calman' curriculum," Journal of Surgical Education, vol. 70, no. 5, pp. 557-562, 2013.

[15] N. Shastri-Hurst, D. N. Naumann, D. M. Bowley, and T. Whitbread, "Military surgery in the new curriculum: whither general surgery training in uniform?" Journal of the Royal Army Medical Corps, vol. 161, no. 2, pp. 100-105, 2015.

[16] T. Singh and J. N. Modi, "Workplace-based assessment: a step to promote competency based postgraduate training," Indian Pediatrics, vol. 50, no. 6, pp. 553-559, 2013. 


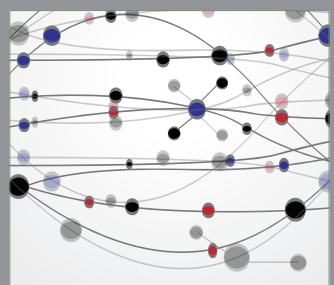

The Scientific World Journal
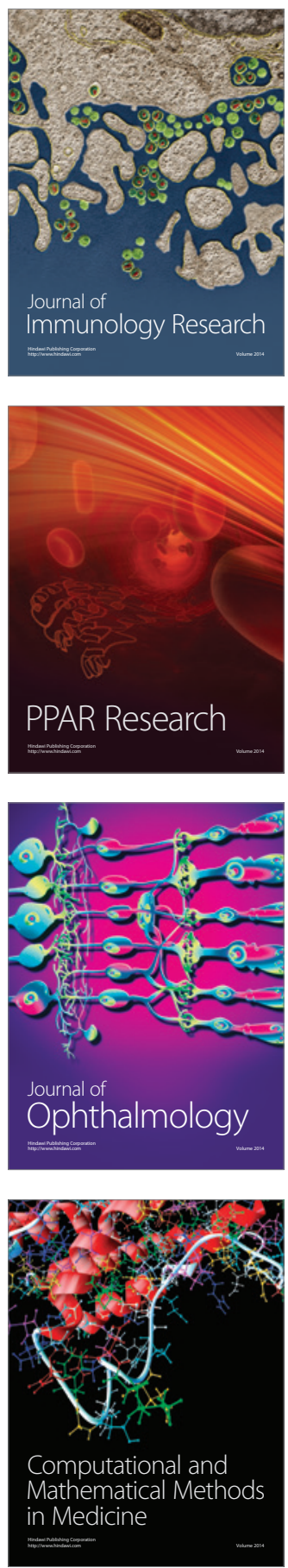

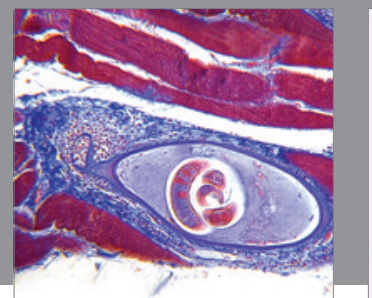

Gastroenterology

Research and Practice
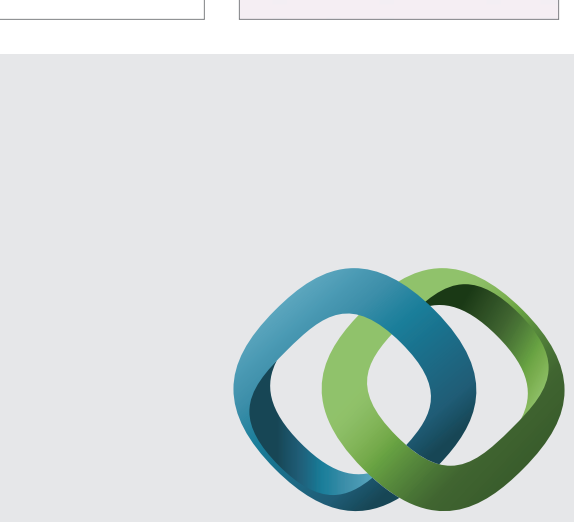

\section{Hindawi}

Submit your manuscripts at

http://www.hindawi.com
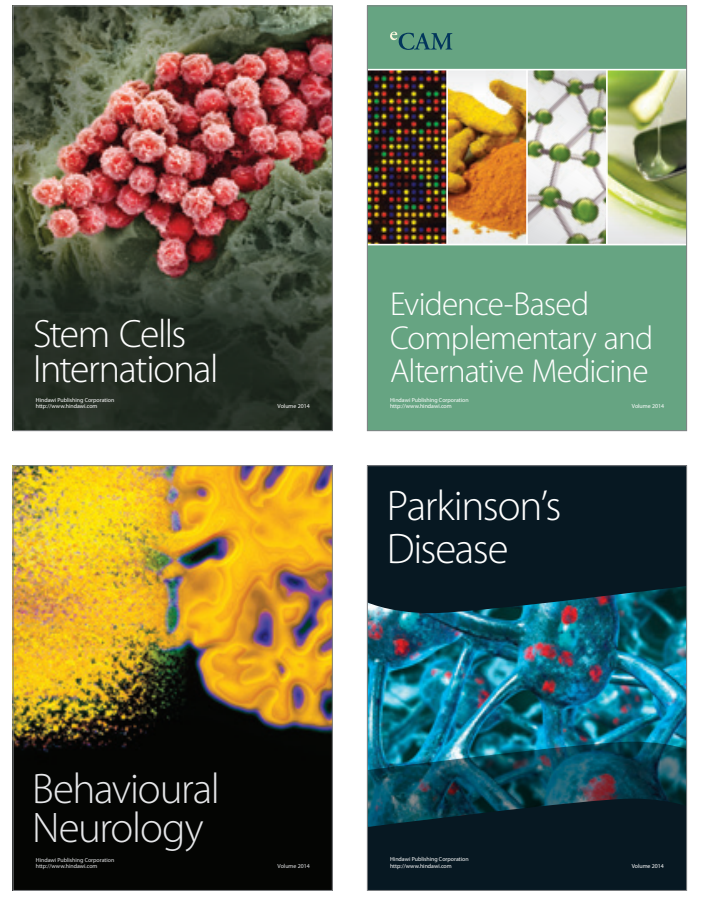
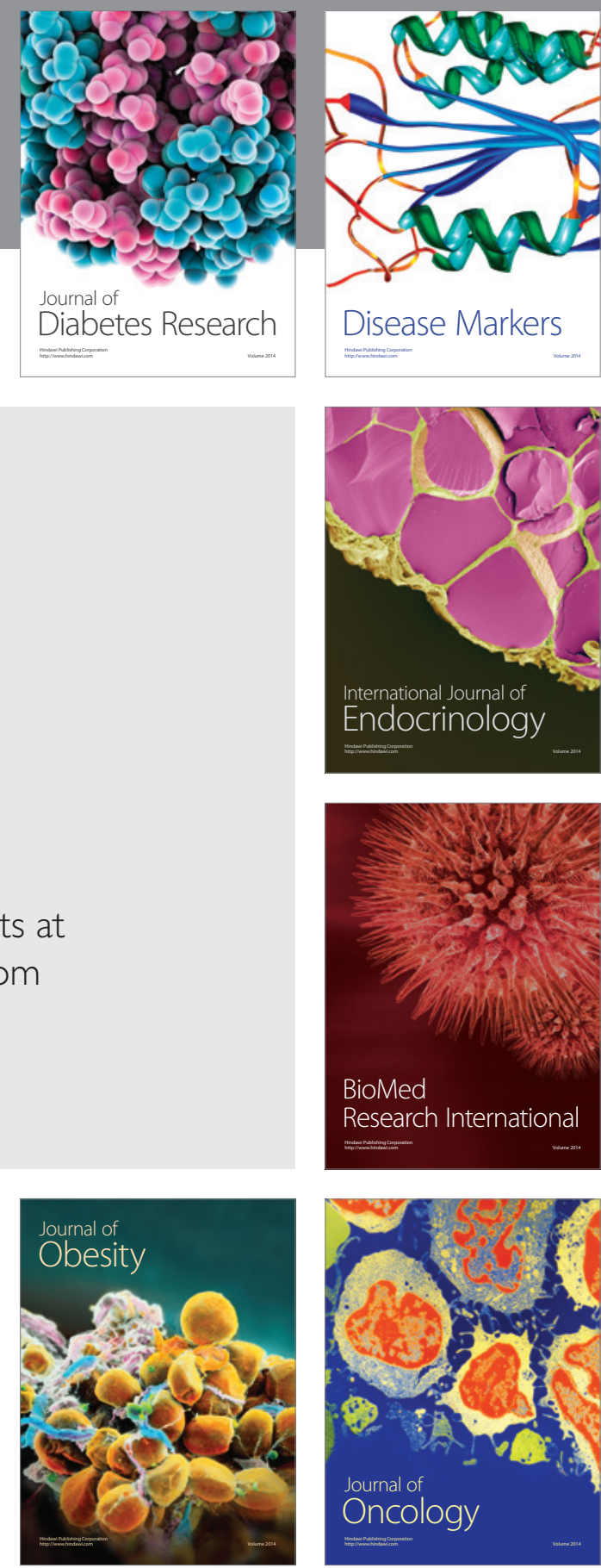

Disease Markers
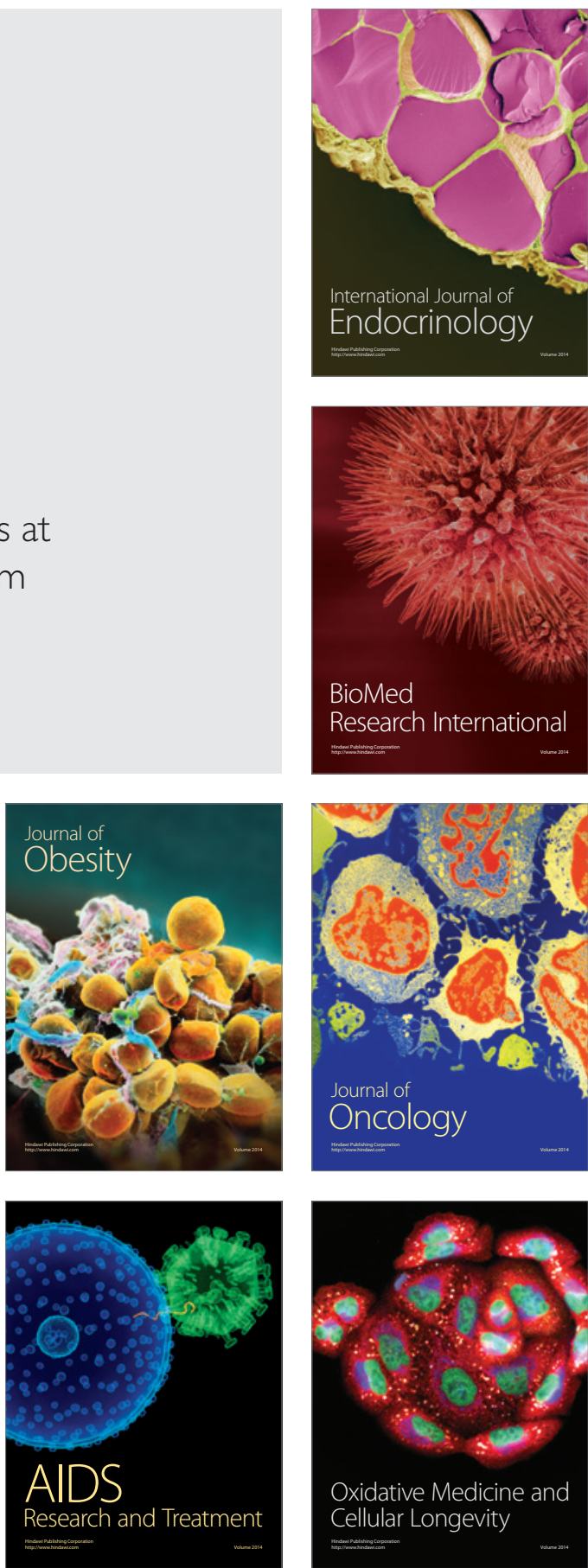